\title{
Whole Systems Thinking and Modelling in the UK
}

\begin{abstract}
UK academic researchers have been vying for a 'whole' systems perspective on energy issues for more than a decade. This research programme has exposed challenges in complex systems thinking and in the dialogue between academic disciplines and epistemic cultures that is needed to mediate the social, technological, and environmental impacts of energy systems. This chapter examines these efforts starting from existing studies that include detailed reports on experiences of interdisciplinary research. By extending these findings via interviews and ethnographic research, this chapter pays particular attention to the role of interdisciplinary computer modelling that was expected to represent complex energy transitions and energy infrastructures of the future. In doing so, this chapter demonstrates how interdisciplinarity has actually worked in three exemplary areas: the diversity of computer models that seek to represent everyday energy demand and how they simplify both demand and other disciplines in so doing; the need for collaborative, cross-cutting research in foresight of future energy scenarios; and how modelling scholars strongly envision their models should become 'useful' for imagined policy and planning stakeholders.
\end{abstract}

Keywords National Centre for Energy Systems Integration (CESI) • UK Research and Innovation (UKRI) • Computer modelling • Modelling evidence $\bullet$ Policy $\bullet$ Ethnography $\bullet$ Social Sciences and Humanities

(C) The Author(s) 2022

A. Silvast, C. Foulds, Sociology of Interdisciplinarity, https://doi.org/10.1007/978-3-030-88455-0_2 


\subsection{INTRODUCTION}

UK academic researchers, assigned to the task by national research funder priorities, have been vying for a 'whole' systems perspective on energy issues for more than a decade. This holistic, and somewhat aspirational, concept of systems has several relevant roots. In particular, the academic field of Systems Engineering has been instrumental in its development, not least because it fundamentally comprises the study and management of whole systems, through the application of interdisciplinary knowledge. It relies on systems theory that emerged in the twentieth century, although it never became a single unified theory of complexity (see Labanca et al. 2020 for further details). Such contributions in recent decades have also evidenced the link between complex systems approaches and the building of energy systems, such as electricity networks and other large infrastructures (Hughes 1983; van der Vleuten 2004).

The concept of whole energy systems is an umbrella term in the UK, evidently developed actively among research funders (e.g. the umbrella organisation UK Research and Innovation, UKRI-previously Research Councils UK, RCUK), research projects (e.g. Transition Pathways consortium and National Centre for Energy Systems Integration, reviewed below), and networks of academics (e.g. UK Energy Research Centre, UKERC). From this starting point, one early designation of whole systems outlines energy research that involves "thinking about all the dimensions of change and drawing on a range of disciplines and expertise" (UKERC 2009, p. 5), where the dimensions are society, economy, and the environment, and the aim is understanding the complex challenges of such holistic energy systems. It situated an interdisciplinary focus that corresponded with a wide definition of an energy system as "the set of technologies, physical infrastructure, institutions, policies and practices located in, and associated with the UK which enable energy services to be delivered to UK consumers" (UKERC 2009, p. 16).

The UK whole systems research programme - of which UKERC's original work was instrumental in summarising-has subsequently exposed new possibilities and challenges in complex systems thinking, as well as stimulated dialogue between academic disciplines and epistemic positions. This chapter is exactly interested in examining these efforts. Indeed, the core empirical focus of this chapter is on the experiences of (mainly Social Scientists) working in a large-scale predominantly technical research project, the National Centre for Energy Systems Integration (CESI). CESI seeks to develop more integrated energy systems in the UK through the 
design of more integrated and interactive 'whole' energy systems for the future. Funded by the UK Engineering and Physical Sciences Research Council (EPSRC), the project involves five research universities and several industrial partners (running 2016-2022). We conducted fieldwork within CESI and in university research groups more generally to access a wider group of energy researchers. ${ }^{1}$

The fieldwork was conducted over two university terms between 2017 and 2018. It included 12 interviews with academic modellers, from mainly two of CESI's UK research universities. By discipline, almost all the interviewees were based in Engineering and the Physical Sciences, although we note that some started their research careers outside of the energy field, in a variety of disciplines, from Architecture and Astrophysics to Applied Mathematics. Three interviewees (25\%) were female, and the rest were male. Nearly all participants were either Postdoctoral or PhD Researchers, and therefore relatively junior members of staff in universities, which we acknowledge is a limitation of the data: more senior staff would have had different perspectives on designing and leading interdisciplinary projects, and early-career researchers are at a known risk from doing interdisciplinary research (Lyall 2019). This empirical shortcoming is mitigated by drawing upon not only the interviews, but also insights from participation in various project events (especially workshops) and from grey project papers - that is to say, we sought insights from situations and materials where senior members of staff may have voiced concerns about interdisciplinarity.

Using original fieldwork and existing reports, this chapter therefore specifically aims to unpack the dynamics of whole systems projects and how they have worked, according to the scholars participating in them. In discussing the experiences of participants in heterogeneous UK research projects, this chapter pays particular attention to the role of interdisciplinary computer modelling (with a specific focus on quantitative planning, operational, and demand tools, as well as qualitative narrative scenarios) that was expected to represent complex energy systems integration, including energy infrastructures of the future.

${ }^{1}$ The first author thanks Prof. Simone Abram, the Principal Investigator of this study at Durham University, for leading the work and conducting some of this field research together. Partly the same materials have been used in our earlier publications (Silvast et al. 2020), but this chapter is a further elaboration in line with the argument of this book focused on interdisciplinary knowledge production. 
This chapter is structured as follows: we begin by contextualising our discussion by drawing key lessons from previous interdisciplinarity-focused evaluations of large UK energy research projects (Sect. 2.2). We then analyse the working practices of CESI in three selected research examples, all of which highlight the need for interdisciplinary research and integration across the Social Sciences and Engineering. The first example is energy demand modelling, the second future scenarios, and the third policy relevance and planning (Sect. 2.3). This chapter concludes by beginning to gather the elements of the framework of this book, which is deepened in the subsequent empirical chapters (Sect. 2.4), on route to its final presentation and discussion in Chap. 5.

\subsection{Reviewing Past Experiences: Interdisciplinary Working Practices in Whole Systems ENERGy RESEARCH}

The UK research projects addressed here have a dual relationship to interdisciplinarity: they have been facilitating interdisciplinary research, but they also produced evaluations of how these disciplinary integrations have worked. These insider reports come from a variety of project contexts and seniorities, indicating the breadth and the diversity of interdisciplinary energy research activities in the UK. While one report examines the experiences of early-career researchers across several UK energy demand research projects, with a particular focus on building research (Mallaband et al. 2017), others were produced as part of particular energy project, such as the first phase of the Transition Pathways to a Low Carbon Economy project ${ }^{2}$ funded by the EPSRC (2008-2012) that interviewed a wide range of academic seniorities (Hargreaves and Burgess 2010; Longhurst and Chilvers 2012). Meanwhile, another report (Winskel et al. 2015 ) examined the whole of UKERC's interdisciplinary capacities and achievements between 2004 and 2014. Across these reports, we contend that there are four common messages that emerge-we now discuss each of these in turn.

\footnotetext{
${ }^{2}$ The Transitions Pathways project was awarded another phase in 2012 to continue under the banner "Realising Transition Pathways-Whole Systems Analysis for a UK More Electric Low Carbon Energy Future" until 2016, a few months before the CESI project started. All the reports used here, in this chapter, were produced in the first phase of the project.
} 
Firstly, interdisciplinarity as a concept and practice found wide support not only among research funders, but also among project participants, whether their broad discipline was in Engineering or the Social Sciences and Humanities (SSH). That said, more salient differences existed with regard to what the participants anticipated from said interdisciplinarity. For example, positions varied from simply gathering interdisciplinary teams in order to broaden perspectives, to more deeply facilitating integrated problem framings - these two positions were linked to different expectations of Engineers and SSH scholars respectively (Hargreaves and Burgess 2010; Longhurst and Chilvers 2012). Consequently, the depth of interdisciplinary collaboration varied in these projects, depending on research output and site, and how exactly to do this disciplinary integration in practice was not always agreed upon (Mallaband et al. 2017).

Secondly, and related to this first message, interdisciplinarity requires an active effort to make a project work coherently for its participants, which is in some tension to how interdisciplinarity should also be about open exploration that should not be made to cohere with ex ante agendas too strongly (Winskel et al. 2015). Moreover, a project's coherence is not a single entity and should not be treated as a simplistic aim. Instead, Longhurst and Chilvers (2012) highlighted four different meanings of coherence in interdisciplinary energy research: the coherent linkage of different knowledge outputs; the comprehensions between project participants; coherence as the credibility of interdisciplinary research outputs; and the more conceptual coherence between different disciplinary worldviews. These forms of coherence also find their corollary in the CESI project examined in the Sect. 2.3.

Thirdly, the early-career researchers in particular (Mallaband et al. 2017), but partly also all the academics interviewed for these reports, highlighted the influence of expectations and roles in the everyday lives of projects. Namely, there are different expectations both concerning what interdisciplinarity is meant to achieve and which roles SSH scholars in particular take and/or are afforded in these projects. This finding joins the insight that has emerged from other fields, such as Synthetic Biology, where one paper (Balmer et al. 2015) recognises no less than nine different roles that SSH scholars can assume in interdisciplinary collaborations. Only one of these roles is a conventional academic research colleague, which highlights that interdisciplinary experts can remain at a greater or 
smaller distance from the core (still disciplinary-based) activity of the said projects. ${ }^{3}$

Fourthly, and importantly for the argument of this book, interdisciplinary working has institutional interdependencies that are recognised by project participants. This means that institutional and research funder support is needed for successful interdisciplinary research-an issue that is not merely about individual achievements among the project members (Mallaband et al. 2017). Part of this problem, as is now well known in interdisciplinarity literature (e.g. Lyall 2019), is that interdisciplinarity is highly favoured as a label but not always served by discipline-based academic reward systems, in terms of, for example, careers, funding, and publications. Indeed, UKERC (Winskel et al. 2015) reiterate the key role that research funding, commissioning, and assessment take in supporting research capacity in interdisciplinarity. They specifically call for collective responsibility of these actors and partnerships among academics, and for them to further pursue these achievements.

In summary, we see that interdisciplinarity requires active maintenance and the (re)production of coherence. We also can observe that the SSH do not always have a preconfigured role in these collaborations, but that this role is instead actively constructed by funding calls and the project participants themselves. The depth and the coherence of interdisciplinary working also vary depending on the kinds of research output or situation at hand.

\subsection{A New Whole Systems Approach and Energy INTEGRATION ISSUES}

In furthering our discussion, this section now turns to in-depth findings from a relevant large-scale interdisciplinary project (CESI), to reflect on how such knowledge production efforts happen in practice, and with what implications for the pursuit of a whole systems' understanding. We begin by detailing the project background and modelling contexts that the CESI project operates within (Sect. 2.3.1), before then discussing three main findings: the diversity of interdisciplinary models and the case of energy demand (Sect. 2.3.2); the challenges of prediction and the consequences

\footnotetext{
${ }^{3}$ The other roles are 'the representative of the public', 'the foreteller', 'the wife', 'the critic', 'the trickster', 'the reflexivity inducer', 'the educator', and 'the co-producer of knowledge'.
} 
of doing interdisciplinarity (Sect. 2.3.3); and interdisciplinary planning under infrastructural conditions (Sect. 2.3.4).

\subsubsection{Modelling Within the National Centre for Energy Systems Integration Project}

The starting point of the CESI project concerned computer modelling in energy. In this, the project was not entirely novel: modelling methods and interdisciplinary discussions on them had been central to various whole systems projects, including both phases of the Transition Pathways to a Low Carbon Economy (Hargreaves and Burgess 2010) and the UKERC Energy 2050 project (UKERC 2009). Computer models are formal representations of bounded energy systems that combine Mathematics and Computing. These tools model envisioned energy systems and can be used to anticipate the energy systems of the future and the present (Silvast et al. 2020).

One well-known model in the UK, although not in use in CESI, is the MARKAL $^{4}$ model (Taylor et al. 2014). The MARKAL model was initiated by the International Energy Agency countries in the 1970s. Within the UK, it was actively maintained and developed by the UCL Energy Systems Team in University College London (UCL 2021). The MARKAL model has been widely used both in the UK and internationally, among governments as well as academics, and for a long period of time until the late 2010s. Its successor model, TIMES, ${ }^{5}$ is very similar. This is how UCL characterises the MARKAL model:

MARKAL portrays the entire energy system from imports and domestic production resources (fossil and renewable), through fuel processing and supply (e.g., refining, bio-processes), explicit representation of infrastructures (e.g., gas pipelines), conversion of fuels to secondary energy carriers (including electricity, heat and hydrogen), end-use technologies (residential, commercial, industry, transport, agricultures, non-energy), and energy service demands (at a sub-sectoral level) for the entire UK energy system. (UCL 2021, n.p.)

By presenting this highly complex energy system by a model, the MARKAL seeks to optimise the system: in practical terms, for example, to

\footnotetext{
${ }^{4}$ MARKAL: MARKet and ALlocation.

${ }^{5}$ TIMES: The Integrated MARKAL-EFOM System.
} 
minimise energy systems costs while constrained by given physical and policy dimensions of energy. The CESI project-which, as already noted, did not develop or use MARKAL — emerged around similar modelling efforts and promised to progress a wide interdisciplinary programme around them. Initially, CESI research noted how current modelling approaches were insufficient, namely because:

[they] are unable to provide sufficiently accurate or detailed, integrated representations of the physics, engineering, social, spatial temporal or stochastic aspects of real energy systems. They also struggle to generate robust longterm plans in the face of uncertainties in commercial and technological developments and the effects of climate change, behavioural dynamics, and technological interdependencies. (UKERC Energy Data Centre 2021, n.p.)

This description links together social and technical aspects of energy and includes a clear need for the Social Sciences (and, more implicitly, Humanities) researchers to engage in envisioning this future integrated system. Therefore, in integrating Engineering and the Physical Sciences to carry out modelling, CESI also ended up employing several SSH scholars, including Economists, Anthropologists, Geographers, and policy-facing energy researchers. An SSH-led stream of enquiry was hence designed into CESI's structure.

While the energy integration terms come from a related background of Systems Engineering (O'Malley et al. 2016), the link between this new energy integration project and whole systems thinking has also been evident. For example, Professor Phil Taylor, the founder and former director of CESI, has noticed a growing "consensus that 'a whole systems approach' is necessary to transform the UK energy system and drive forward the government's industrial strategy" (quoted in Northern Gas Networks 2017).

Sections 2.3.2, 2.3.3, and 2.3.4 recount how scholars working towards bringing about these 'whole systems'-in particular inside CESI, but also in related project environments-developed tools and methods for integrating academic disciplines and saw the possibilities and limits of their tools in bringing this integration about. Particular attention is given to computer models as means by which the interdisciplinary whole systems are being enacted. 


\subsubsection{The Diversity of Interdisciplinary Models and the Case of Energy Demand}

The first key finding from the ethnography of whole systems modelling concerned just how different computer models are, and that there are various subgroups of modelling scholars that bring these differences about. In a general sense, this observation is not novel. The literature on energy research has provided numerous classifications of 'modelling families' in energy, which comprise different approaches, academic fields, goals, and differences among bottom-up and top-down models ( $\mathrm{Li}$ et al. 2015; Pfenninger et al. 2014). However, what is novel here is bringing this diversity of models to the question of interdisciplinary knowledge production: what does the well-recognised diversity among energy models imply for integrating academic disciplines in knowledge production with models?

To enable our following discussion to be more tangible, and less abstract, we now use a seemingly straightforward topic as our object of enquiry: energy demand. In this modelling setting, demand is usually considered as final energy consumption. Nevertheless, as another large UK interdisciplinary energy consortium, CREDS, ${ }^{6}$ has described it, energy demand is a wide topic. Demand is regarded as comprising all human activity that depends on available energy, shaping energy infrastructure as a result. Accordingly, demand "drives the whole energy system, influencing the total amount of energy used; the location of, and types of fuel used in the energy supply system; and the characteristics of the end use technologies that consume energy" (CREDS 2021 no pagination).

This much is clear to the current social scientific works on demand and everyday practices (e.g. Shove and Trentmann 2018). But how can energy modellers represent this demand with their quantitative tools? A recent UKERC working paper (Hardt et al. 2019) examined the energy models that inform UK government energy policy and enquired how these mainstream models represent energy demand. They generated a range of findings, with 13 core models selected covering different approaches, purposes, and parts of the energy system. While the model types also differed-ranging from econometric to system-optimisation, economic, and sector-based models - they clearly shared an emphasis on providing large technological detail as the main output. However, all the reviewed models also showed "limitations with regard to the representation of non-technological drivers

${ }^{6}$ CREDS: Centre for Research into Energy Demand Solutions. 
of energy demand" (Hardt et al. 2019, p. 6). It was especially apparent that drivers of demand (e.g. behavioural, economic, and social dynamics) were both treated as exogenous assumptions in the models and did not belong to the objective of the modelling. This is interesting insofar as the studied modellers saw that their discipline could not offer any proof on demand, but this also made the studied models inadequate for understanding how demand is constituted and could change.

The report (Hardt et al. 2019) did not study academic modelling per se-as they focused only on how academic models are used in UK policy processes-but they did subject considerable expectation to interdisciplinary academic work that could fill the knowledge gap on demand. The authors anticipate that:

there is still considerable scope for energy models to provide better representations of demand-side energy policies, especially with regard to nontechnological aspects. The academic literature contains some promising attempts of incorporating more realistic representations of social and behavioural processes in energy models. (Hardt et al. 2019, p. 6)

This quote closely resembles the problem that the first author was asked to address in June 2018, with a few weeks to work on it before a project workshop: namely, to explore how models in Energy Systems Engineering could become more sociotechnical, by including qualitative and quantitative information on demand for energy. This was not an unknown problem to CESI. It involved a whole research group of 'demand modellers', that is, modellers dealing with everyday energy demands, and who had in many ways crossed over to the SSH. We saw these researchers actively exploring and using sociological practice theories in their talks, for instance, and seeking to complement their models with our 'Social Science data' on everyday life to make the results more uncertain or what was termed as 'fuzzy' and on occasions, even labelling their work as explicitly social scientific, or as the looser common term in use goes, 'sociotechnical' (cf. Love and Cooper 2015).

For example, the first author attended a project meeting where one of the CESI demand modellers named his presentation as "socio-technical energy demand modelling". The presenter's research question asked how people and institutions are represented in energy systems models, and the presentation itself travelled from qualitative narrative scenarios to modelling of behaviour, the rise of active energy citizens, and how social 
practices confront abrupt changes in societies. While the speaker remained ambivalent on his discipline being a Social Science, in the discussion that ensued, the contribution of the presentation was explicitly addressed as social scientific by some external participants of the workshop.

However, while CESI did have a team of demand modellers, this did not mean that there was a single way to accomplish this integration of demand to modelling. As the demand modellers themselves clearly understood, modelling demand had to confront considerable uncertainties surrounding everyday practices. Indeed, as one CESI modeller observed:

\begin{abstract}
[In a] Traditional model you put discrete information about the control of the system, something comes on at certain time, goes off at a certain time, you can play about with temperature settings and so on, but there is discrete information ... The fuzziness of what happens, lots of people using those things where they might start this time, or they might start this time, or this time... It's a bit difficult to put in manually into this model. [Man, 40-49 years, Senior Lecturer in Energy)
\end{abstract}

More than saying that some models could not appropriate uncertain demand-which was true for some, but not all of them-it was more accurate that demand, in itself, had 'interpretative flexibility' (Pinch and Bijker 1984). Different scientists offered not merely different interpretations of what demand may be, but also different designs for how demand could be included in their models. An explorative classification, based on the fieldwork, found these families of demand approaches in CESI:

1. The demand curve approach: demand is treated as external to the modelled energy system, but interacts with it as its 'environment' or 'input'. This demand can be represented, for instance, by actual long-term measurement on energy use. This is close to the models identified (Hardt et al. 2019) that also use demand as exogenous to the model.

2. The known demand approach: demand is already 'known' by the model. It can be represented by actual energy use data or simulated by an algorithm. This 'known' constant of the model is fixed, where other variables (e.g. voltage and temperatures) are solved by the model. The difference to the demand curve approach is that, here, demand is not exogenous, but is internal to the modelled system. 
3. The techno-economic approach: demand is represented by assuming consumers are rational economic actors that, for example, respond to price signals. This assumption exists behind many future planning models and considerations on introducing dynamic (e.g. time-ofuse) electricity pricing to households. It is important to note that many models assume these rational consumers to actually exist, even if this assumption has been widely challenged in the $\mathrm{SSH}$ for decades (cf. Christensen et al. 2020).

4. The demand modelling approach: demand becomes the actual output of the modelling work. This could happen by measuring it empirically in households or simulating it, or often via a combination of both. This approach persists, for instance, in many building modelling studies where modellers seek to know how energy demand evolves in certain kinds of buildings and with a set of improvements to, for example, energy efficiency.

5. The impacts to demand approach: the model results, whether the models include demand or not, will always enact certain kinds of energy technologies and energy systems, often in a simulated representation. This approach to demand means enquiring what those changed technologies and systems would imply for the everyday demand of people and the activities and habits that constitute it.

6. The demand foresight approach: especially when it comes to anticipating future energy systems, it is important to know how society's demands for energy may change in the coming decades, for example, up to 2050 in line with governmental decarbonisation targets. This approach to demand is common in scenarios and storylines that merge 'qualitative' with 'quantitative' modelling, which will be discussed more in Sect. 2.3.3.

While overlapping and related to one another, these approaches to the concept of demand are not the same, and there may be no middle ground that would integrate all of them. To a varying extent, most of them clearly call upon integration from the SSH to produce insights on people's activities that constitute demand. But depending on which approach to demand is taken, the concepts, methodologies, and evidence bases from $\mathrm{SSH}$ would be almost entirely different. The provision of more accurate demand curves might call expertise from, for example, the Statistics and Economics disciplines of SSH. Whereas enquiries on how new energy systems will affect people's lives might need insights and tools from field methods in 
Anthropology and Sociology; or Law and Policy Studies when the legal and regulatory dimensions of these changes are of interest. Further, the questions relating to how our societies will develop in 2050 could be addressed by forecasting methods. Such different contributions would ultimately mean entirely different tasks for SSH engagement in modelling projects.

The above findings also suggest that modelling-while largely based across the disciplines of Engineering, Economics, Statistics, and Computer Science, and in itself is firmly interdisciplinary-cannot be unified as a single (inter)discipline in its own right. The notion of a discipline remains important for this interdisciplinary energy work, especially when scholars attribute what belongs to their expertise and what does not, which is typical when considering demand and human activities. But disciplines do not entirely capture the different ways in which modellers produce knowledge, again, for example, about demand. Resembling 'epistemic cultures' (Knorr Cetina 1999), different modellers had various tools and methods, types of reasoning, ways to establish evidence, and ideals concerning theory-empirics fits. These differences extended to how their models could integrate demand, including reflections on to what extent it was even possible. Those engaging in interdisciplinary collaborations with modellers should pay attention to these differences as much as the disciplinary differences, for example, between core Engineering and $\mathrm{SSH}$, which are communicated far more often in our experience.

Yet another conclusion to be made from the findings above is just how distinctly the modellers and policy studies of models attributed the role of $\mathrm{SSH}$. If we assume that demand is the domain of the SSH-and this was routinely assumed by the modelling experts and we have never heard it questioned by them-then it is appropriate to ask, which SSH scholars would recognise that they study, for example, 'non-technological drivers of demand' or 'social and behavioural processes'? The answer is that these are labels attributed to the SSH from the outside. However, the issue runs deeper than the process of labelling, because these labels are symptoms of wider institutional norms and deeper-running expectations. As such, SSH scholars cannot (or, rather, should not) aim to simply replace uncomfortable labels with more appropriately deemed alternatives.

Here, we can draw on work positioned between Anthropology and Information Science, to discuss a more significant knowledge gap (Forsythe 1999). The idea that patterns of behaviour and organisation can be integrated to computer models also assumes that those patterns are out there, 
just waiting to be detected by SSH-led observation. Yet, the issue is that many current SSH methodologies do not provide answers to such a problem. It is now well known that topics (e.g. behaviour and organisation) are not just 'out there' in the social world, but constructed during the research process, and our methods not only describe them but also bring them about, often during a meticulous and long research process (Gobo 2008; Silvast and Virtanen 2019). This makes it challenging to work in interdisciplinary modelling projects partly in hidden ways. If the research problems have been designed in such a way where an appropriate SSH response is difficult, this might require engaging the partners on what SSH exactly is and is not, which is very rarely (if ever) among the deliverables of common research and innovation projects.

\subsubsection{The Challenges of Prediction and the Consequences of Doing Interdisciplinarity}

A second key finding, where the role of interdisciplinary working becomes manifested, concerns foreseeing the energy systems of the future. This is the core domain of large energy research projects, with the European Union and several national governments having set decarbonisation goals up to 2050 , and researchers seeking sociotechnical solutions to help reach those goals. Many computer models, although not all of them, are committed to foreseeing how this future energy system will come about. Earlier relevant UK projects, such as the Transition Pathways (Longhurst and Chilvers 2012), were explicitly committed to scoping this complex sociotechnical change in an interdisciplinary manner. This was also true of CESI, whose main research aim was to understand both future energy supply and demand.

The notion of predicting energy futures has also become increasingly problematised, which modellers themselves know much about. Here, the underlying issue is uncertainty and including it in the modelling process in a useful way. Indeed, past academic and policy predictions have had demonstrable issues in dealing with uncertainty, and are thus starting points for considering improvements. For instance, a UKERC retrospective study of UK energy forecasts discovered past models to have been poor at incorporating future uncertainties, and many real-world events that had followed these predictions would have been considered to be too extreme when making the predictions (McDowall et al. 2014). 
Most of the studied future scenarios had only emphasised economic issues (e.g. predicting oil prices) and focused on specific technologies (e.g. nuclear power). They were focused on cost-optimisation and none of the examined scenarios would allow for significant institutional changes (McDowall et al. 2014). Indeed, institutional arrangements, political decisions, and the impacts of societal actors may be difficult to include in a useful modelling process, although some influential attempts exist nonetheless ( $\mathrm{Li}$ et al. 2015). A problem related to this is that energy models are not modelling a fixed target system. Instead, the energy systems being modelled-including their regulations, technologies, business models, and end-use practices - are undergoing change simultaneously (McDowall 2014). This situation challenges the prospects of predicting how those systems will behave in the future.

The general response to these issues of forecasting has been to reformulate the aim: towards aspiring for 'good-quality', rather than 'accurate', predictions of the future. We have come far from the scenario-building and forecasting exercises of the 1970s and the 1980s, where calculative models would be used to make deterministic energy supply policies and were even performative to the notion of what energy policy is all about (Aykut 2019). Today's academic energy futures are rather named as storylines - that is, narrative stories and visions (Fortes et al. 2015) —and there is an explicit assumption that storylines are not predictions or forecasts, and should not be treated as such.

Typically, storylines designate that certain events will happen in the future, such as "macroeconomic and microeconomic policies simultaneously stimulate innovation, creativity, and technological improvement" (Fortes et al. 2015, p. 164). Yet, the scenario method does not actually predict that this will happen; in some cases and depending on the method, it does not claim it is more likely, nor does it have to claim that it is more favourable than any another envisioned scenario, such as the innovation not being stimulated. The starting point is rather in developing storylines towards challenging decision-makers, and these stories can also be linked with information from quantitative models in a myriad of ways.

CESI was also similarly exploring qualitative and quantitative scenarios with the aim of improving strategic insights for energy systems integration. To this aim, it set about developing narrative scenarios for the UK. At the time of writing this, the scenarios are a work-in-progress and it is not on our agenda to comment more on effective scenario design or different approaches to scenarios, but we do draw on a grey paper including several 
CESI members (Wheatcroft et al. 2019). We will simply note that the CESI approach to scenarios seems to resemble what was outlined above, especially the impossibility of perfect prediction of future events and scepticism when it comes to applying probabilities to future scenarios. Rather than predicting, the paper by CESI and others outlines five aims for a scenario (Wheatcroft et al. 2019, p. 6):

1. Plausible: a scenario should be plausible and come with a narrative justifying each event or change in the underlying assumptions.

2. Distinctive: the different scenarios should be distinctive enough in terms of the key factors for there to be a clear difference between them.

3. Consistent: interaction between key factors should be taken into account. For example, macroeconomic factors may impact important aspects of the scenarios simultaneously.

4. Relevant: each scenario should be relevant in terms of giving a specific insight into the future (e.g. the government increases spending on green projects and subsidies).

5. Challenging: scenarios should challenge the conventional view on things that may affect the project in question.

This listing moves us back to our interest in interdisciplinary working practices. The authors of the CESI paper are particularly interested in mainstream mathematical and statistical modelling and their relationships with scenarios. However, what role will SSH play in formulating the scenarios of the future?

Here, we notice a very different pathway depending on whether scenarios are meant to be predictions or not. If scenarios are just like predictions, then SSH research can be deployed to discover whether the predictions seem to be realistic or not, and why. Economists and Social Scientists could work together to find out how likely, for example, it is that macroeconomic policies work in stimulating innovations in different cases, and how that may be changing. However, the problem, as the writers of this aforementioned CESI paper (Wheatcroft et al. 2019) see it, is that scenarios and storylines are not meant to be predictive, and there is a delicate (and not adequately communicated) difference between predicting and simply offering plausible explanations. But, what would this difference mean in operational terms? Here, more work is needed to explain the terms in a way that clarifies the difference to various involved disciplines. 
We also see a risk that the SSH become an add-on that merely challenges the scenarios developed by other disciplines (Robison and Foulds 2019), but this does not yet constitute strong interdisciplinary integration. We would claim that to take the five recommendations above to their logical conclusion-to form scenarios that are plausible, distinctive, consistent, relevant, and challenging-requires SSH scholars to be integrated into the work of forming scenarios from its start, which was always the case in CESI. Enquiring what is, for example, plausible about energy futures is a complex task and requires different expertise across disciplines, including various kinds of SSH scholars, from Anthropology to Philosophy, Ethics, Political Science, and beyond (cf. Ialenti 2020). Here, we again voice the need for going beyond simple prescriptions, such as SSH scholars only study the 'non-technological drivers of demand'. Adequate and balanced disciplinary representation (Winskel et al. 2015) is needed to ensure that energy scenarios' definitions of the future are not shifted towards biases developed by other dominant disciplines.

\subsubsection{Interdisciplinary Planning Under Infrastructural Conditions}

Our third finding was that more than 'accuracy' or 'prediction', the CESI modellers were visibly more interested in how their models are used and by whom. This became especially pronounced in the contexts of policy, politics, and planning - contexts set out with the borders of the computer models, yet meant to be informed by modelling results (Silvast et al. 2020). In contrast to the two other areas mentioned in the previous two sub-sections - where the SSH were often implicitly present, by topics that were assumed to be SSH-relevant, but were not always mentioned by name-in this case, the role of SSH was more explicit and pronounced.

Herein, we will use a workshop report, published by Centre for Digital Built Britain scoping network, called Planning Complex Infrastructure Under Uncertainty, and including several CESI members, as an exemplar (Dent et al. 2019). ${ }^{7}$ At its outset, the report summarises the work of "researchers in mathematical sciences, engineering and social sciences" (Dent et al. 2019, p. 1). In addition, further than this, the report's one

\footnotetext{
${ }^{7}$ For disclosure, the first author was one of the workshop participants, took part in the discussions, and is named in the report.
} 
core recommendation was to involve both $\mathrm{SSH}$ in the planning of infrastructures. The report states this aim:

There is a need to incorporate social science (including humanities) research around issues such as understanding 'value', capturing change in value/s, multiplicity of voices (success for whom?), and critical assessments of data and models. There are different potential relationships available between social science research and that of science, mathematics and engineering, and scope to consider these relationships creatively in developing interdisciplinary work; there is value in social science research not only to support and/or challenge work in technical subjects but also sometimes to lead or shape the challenges addressed and approaches taken. (Dent et al. 2019, p. 4)

There is much packed into this quote. It starts by designating certain implicitly 'non-technical' tasks for SSH, such as increasing the diversity of values and voices in planning infrastructures, and critiquing the models and data prepared presumably by non-SSH disciplines. But the end of the quote shows considerably more variation to the relationships between disciplines. It even envisions that, in some cases, the disciplinary balance could be turned the other way, to let SSH scholars shape research projects on infrastructural matters.

Another topic where the SSH are more implicitly present comes to the policy relevance of modelling tools. While focused around the topics of modelling techniques and research, the report is also written in a way that it clearly seeks to translate the modelling practice into new areas. A particular reliance is placed on models that support decision-making and that are even designed with decision-support in mind. As the authors express this aim:

It is important to guard against matters such as collecting data for the sake of having a large dataset, or confusing optimality in the model world with a good decision in the real world - the real goal being to identify decisions which one has logical reason to believe are good ones in the real world. (Dent et al. 2019, p. 9)

In our ethnography, we observed a widely shared similar interest: in decision-making, decision-support, and the 'appropriate' use of models by these decision-makers. Curiously, however, this role of decision-support is not where the SSH were recognised above. The report still states a division where the SSH predominantly study the 'non-technical', and perhaps 
also the 'non-political' aspects of energy, or study the political insofar as it is translated into values and opinions. Not mentioning decades of SSH insights on governance, political studies, and policy analysis, the model designers appropriated the needs of the model 'end-users' on their common sense: using assumptions about how 'policy decisions' are made, rather than in-depth knowledge of governance practices. As we have suggested elsewhere (Silvast et al. 2020), training in governance would be an appropriate step for modellers working in energy-policy-interfaces and seeking to engage policymakers. A further integration of models and policy would also require the existence of models that can act as 'boundary objects' (Star and Griesemer 1989)—such as the popular MARKAL model in the UK, which is understood across different social worlds (e.g. academia and energy policy) and has hence become widely deployed (Taylor et al. 2014). All of this shows that the integration of the social worlds of policy, SSH, Engineering, and so on is not a simple task and requires more than explicit proclamations of interdisciplinary work to happen successfully.

\subsection{Conclusions}

This first empirical chapter of the book studied UK whole systems energy modellers and scientists: scholars who had explicitly set out to integrate natural, environmental, social, and technical disciplines, in coming to more relevant solutions to current energy issues.

The first main section (Sect. 2.2) of this chapter recounted how the issues of this research have been known, and documented, for a decade, often by reports from these projects from within. The next main section (Sect. 2.3) sought to study aligned themes in one of the most recent whole systems research programmes in the UK: the National Centre for Energy Systems Integration (CESI). CESI had designed SSH into its enquiry, but relied strongly on energy computer modelling in conjunction with interdisciplinary SSH research. Based on an ethnography conducted within the CESI and relying on the fieldwork and grey papers published by the CESI members, we travelled through three findings. The first finding was the diversity of modelling approaches and resulting variety of SSH integrations within these, using the case of energy demand modelling across the CESI and in UK policy more generally (Sect. 2.3.2). The second finding looked at how energy futures are being envisioned by interdisciplinary energy projects and what role SSH scholars could play in constructing a 
balanced view of these futures (Sect. 2.3.3). The final finding examined how CESI itself had vied for including SSH in planning complex infrastructures under uncertainty, and what that had implied for the possibilities of other disciplines to work, for example, on the policy relevance of the modelling results (Sect. 2.3.4).

We now summarise a set of six lessons learned from this first empirical chapter, which we hope further signals our direction of travel, on route to our end-of-book synthesis where we outline our Sociology of Interdisciplinarity framework. Our first lesson: UK research funding has had significant effects in bringing about more interdisciplinary agendas and certain kinds of working practices and collaborations when it comes to interdisciplinary working (e.g. Winskel et al. 2015). While we cannot study the structuring impact of funding with the data that we have herefor example, for scientific productivity (Goldfarb 2008) —we do argue that the research of individual scholars and groups is organising around themes that are of high priority in addressing grand societal challenges when it comes to energy (Royston and Foulds 2021). This chapter has started to document how individuals and groups working on these interdisciplinary projects develop joint work addressing the themes in their own ways and contexts. This is a theme we will continue to develop throughout the book.

Second, it is useful to think about interdisciplinary knowledge production in specific epistemic cultures (Knorr Cetina 1999), by which we mean knowledge-oriented cultures of scholars that cut across broad academic disciplines, such as Engineering, Physics, and the SSH. This view helps point out that disciplines-while highly relevant for reasons we point below-are not quite complex enough as units of analysis for understanding, for example, the diversity of knowledge production tools that (energy) researchers use. An example is the considerable variety that exists within energy modelling.

Third, the epistemic cultures in interdisciplinary projects are mediated by specialised boundary objects (Star and Griesemer 1989), such as computer models, conceptions of energy demand, or energy scenarios, as studied in this chapter. Boundary objects are artefacts, concepts, or methods that lie at the interface of different social worlds, such as politics and the academia. Because their identity is understood across these worlds-even in cases where they lack a proper definition-they enable co-operation and coordination between them. For instance, this comes very close to how the modellers examined in this chapter hoped that their models would work: as an object by which SSH scholars, Engineers, Mathematicians, 
policymakers, and various other actors alike could interact across their social worlds, even without always understanding the intricacies of how the models work.

Fourth, we see not only co-operation between different disciplines, but dynamics of appropriation. Interdisciplinary projects can see one discipline appropriating the tools and methods of other disciplines. This appropriation does not have to imply power dynamics or one discipline being more powerful than the other, and it does not have to be embedded in resource distribution (although it can be). Instead, in our observations, appropriation happened in much more mundane ways, especially relating to the labelling of what SSH do in modelling projects, without consulting what their research designs can actually allow. This generates the view that SSH only study, for example, 'social and behavioural processes', thereby implying activities that few SSH scholars would perhaps recognise and which would require much more demanding research resources than may be given to them for project implementation.

Fifth, while interdisciplinarity is often favoured by funding bodies and researchers as a label, this conceals the considerable interpretative flexibility of the concept itself. This premise extends to how those working in interdisciplinary projects interpret certain important interdisciplinary concepts (e.g. scenarios, energy demand, and energy policymaking). This finding is an extension of boundary objects, but makes a different conclusion: when scholars interpret concepts differently (e.g. energy demand), it means not only that the meaning differs, but that they would design technologies (e.g. demand modelling) in a distinct manner. This has major implications for how academic disciplines can work together for studying such concepts.

Sixth, our final lesson from this chapter: even in contexts where interdisciplinary is highly valued as an explicit strategy of the funding body, we should pay close attention to the continued importance of conventional academic disciplines in interdisciplinary work. There are more general reasons for the continued importance of disciplines, such as traditions of research offering coherence and presupposed practices, and students being taught in their paradigmatic instruments (Michael 2017). But the matter is also manifest in mundane project life and outputs: our materials show scholars routinely referring to broad academic disciplines as one homogenous entity and thereby with reference to a singular name (e.g. Mathematics, Statistics, Engineering, and Social Sciences), as part of them making sense of their own work in relation to others (Foulds et al. 2017). 
We must take this continued use of disciplines by name seriously, since they clearly still mean much even for those engaged in projects that are meant to crossdisciplinary boundaries.

\section{REFERENCES}

Aykut, S.C., 2019. Reassembling energy policy: Models, forecasts, and policy change in Germany and France. Science and Technology Studies 32, 13-35. https://doi.org/10.23987/sts.65324

Balmer, A.S., Calvert, J., Marris, C., Molyneux-Hodgson, S., Frow, E., Kearnes, M., Bulpin, K., Schyfter, P., MacKenzie, A., Martin, P., 2015. Taking roles in interdisciplinary collaborations: Reflections on working in post-ELSI spaces in the UK synthetic biology community. Science and Technology Studies 28, 3-25. https://doi.org/10.23987/sts.55340

Christensen, T.H., Friis, F., Bettin, S., Throndsen, W., Ornetzeder, M., Skjølsvold, T.M., Ryghaug, M., 2020. The role of competences, engagement, and devices in configuring the impact of prices in energy demand response: Findings from three smart energy pilots with households. Energy Policy 137, 111142. https://doi.org/10.1016/j.enpol.2019.111142

CREDS, 2021. What is energy demand [WWW Document]. URL https://www. creds.ac.uk/what-is-energy-demand/ (accessed 5.30.21).

Dent, C., Anyszewski, A., Reynolds, T., Masterton, G., Du, H., Tehrani, E., Lovell, K., Mackerron, G., 2019. Planning complex infrastructure under uncertainty-Network final report. https://doi.org/10.17863/CAM.40455.

Forsythe, D.E., 1999. "It's just a matter of common sense": Ethnography as invisible work. Computer Supported Cooperative Work 8, 127-145. https://doi. org/10.1023/A:1008692231284

Fortes, P., Alvarenga, A., Seixas, J., Rodrigues, S., 2015. Long-term energy scenarios: Bridging the gap between socio-economic storylines and energy modeling. Technological Forecasting and Social Change 91, 161-178. https://doi. org/10.1016/j.techfore.2014.02.006

Foulds, C., Robison, R., Balint, L., Sonetti, G., 2017. Headline reflectionsSHAPE ENERGY Call for Evidence. Cambridge.

Gobo, G., 2008. Doing ethnography. Sage, London.

Goldfarb, B., 2008. The effect of government contracting on academic research: Does the source of funding affect scientific output? Research Policy 37, 41-58. https://doi.org/10.1016/j.respol.2007.07.011

Hardt, L., Brockway, P., Taylor, P., Barrett, J., Gross, R., Heptonstall, P., 2019. Modelling demand-side energy policies for climate change mitigation in the UK: A rapid evidence assessment. UKERC, London. 
Hargreaves, T., Burgess, J., 2010. Pathways to interdisciplinarity: A technical report exploring collaborative interdisciplinary working in the Transition Pathways consortium, Working Paper-Centre for Social and Economic Research on the Global Environment. University of East Anglia, Norwich.

Hughes, T.P., 1983. Networks of power: Electrification in Western Society, 1880-1930. Johns Hopkins University Press, Baltimore.

Ialenti, V., 2020. Deep time reckoning: How future thinking can help Earth now. MIT Press, Cambridge, MA.

Knorr Cetina, K., 1999. Epistemic cultures. Harvard University Press, Cambridge, MA. https://doi.org/10.2307/j.ctvxw3q7f

Labanca, N., Pereira, Â.G., Watson, M., Krieger, K., Padovan, D., Watts, L., Moezzi, M., Wallenborn, G., Wright, R., Laes, E., Fath, B.D., Ruzzenenti, F., de Moor, T., Bauwens, T., Mehta, L., 2020. Transforming innovation for decarbonisation? Insights from combining complex systems and social practice perspectives. Energy Research and Social Science 65. https://doi. org $/ 10.1016 /$ j.erss.2020.101452

Li, F.G.N., Trutnevyte, E., Strachan, N., 2015. A review of socio-technical energy transition (STET) models. Technological Forecasting and Social Change 100, 290-305. https://doi.org/10.1016/j.techfore.2015.07.017

Longhurst, N., Chilvers, J., 2012. Interdisciplinarity in transition? A technical report on the interdisciplinarity of the Transitions to a Low Carbon economy consortium. University of East Anglia, Norwich.

Love, J., Cooper, A.C.G., 2015. From social and technical to socio-technical: Designing integrated research on domestic energy use. Indoor and Built Environment 24. https://doi.org/10.1177/1420326X15601722

Lyall, C., 2019. Being an interdisciplinary academic, being an interdisciplinary academic. Palgrave Macmillan, London. https://doi. org/10.1007/978-3-030-18659-3

Mallaband, B., Wood, G., Buchanan, K., Staddon, S., Mogles, N.M., GabeThomas, E., 2017. The reality of cross-disciplinary energy research in the United Kingdom: A social science perspective. Energy Research and Social Science 25, 9-18. https://doi.org/10.1016/j.erss.2016.11.001

McDowall, W., 2014. Exploring possible transition pathways for hydrogen energy: A hybrid approach using socio-technical scenarios and energy system modelling. Futures 63, 1-14. https://doi.org/10.1016/j.futures.2014.07.004

McDowall, W., Trutnevyte, E., Tomei, J., Keppo, I., 2014. UKERC energy systems theme reflecting on scenarios. UKERC, London.

Michael, Mike., 2017. Actor-network theory: Trials, trails and translations. Sage, London.

Northern Gas Networks, 2017. Northern gas networks and CESI launch unique gas and whole systems research laboratory-IntEGReL [WWW Document]. URL https://www.northerngasnetworks.co.uk/2017/01/26/northern-gas- 
networks-and-cesi-launch-unique-gas-and-whole-systems-research-laboratoryintegrel/ (accessed 5.30.21).

O'Malley, M., Kroposki, B., Hannegan, B., Madsen, H., Andersson, M., William, D., Mcgranaghan, M.F., Kroposki, B., Hannegan, B., Madsen, H., Andersson, M., Dent, C., 2016. Energy systems integration: Defining and describing the value proposition, Nrel/Tp-5D00-66616.https://doi.org/10.2172/1257674

Pfenninger, S., Hawkes, A., Keirstead, J., 2014. Energy systems modeling for twenty-first century energy challenges. Renewable and Sustainable Energy Reviews 33, 74-86. https://doi.org/10.1016/j.rser.2014.02.003

Pinch, T.J., Bijker, W.E., 1984. The social construction of facts and artefacts: Or how the sociology of science and the sociology of technology might benefit each other. Social Studies of Science 14, 399-441. https://doi. org/10.1177/030631284014003004

Robison, R., Foulds, C., 2019. 7 principles for Energy-SSH in Horizon Europe: SHAPE ENERGY Research \& Innovation Agenda 2020-2030. Cambridge.

Royston, S., Foulds, C., 2021. The making of energy evidence: How exclusions of Social Sciences and Humanities are reproduced (and what researchers can do about it). Energy Research and Social Science. https://doi.org/10.1016/j. erss.2021.102084

Shove, E., Trentmann, F., 2018. Infrastructures in practice, infrastructures in practice. Routledge, London. https://doi.org/10.4324/9781351106177

Silvast, A., Laes, E., Abram, S., Bombaerts, G., 2020. What do energy modellers know? An ethnography of epistemic values and knowledge models. Energy Research and Social Science 66, 101495. https://doi.org/10.1016/j. erss.2020.101495

Silvast, A., Virtanen, M.J., 2019. An assemblage of framings and tamings: Multisited analysis of infrastructures as a methodology. Journal of Cultural Economy 12, 461-477. https://doi.org/10.1080/17530350.2019.1646156

Star, S.L., Griesemer, J.R., 1989. Institutional ecology, 'translations' and boundary objects: Amateurs and professionals in Berkeley's Museum of Vertebrate Zoology, 1907-39. Social Studies of Science 19, 387-420. https://doi. org/10.1177/030631289019003001

Taylor, P.G., Upham, P., McDowall, W., Christopherson, D., 2014. Energy model, boundary object and societal lens: 35 years of the MARKAL model in the UK. Energy Research and Social Science 4, 32-41. https://doi.org/10.1016/j. erss.2014.08.007

UCL, 2021. UK MARKAL [WWW Document]. URL https://www.ucl.ac.uk/ energy-models/models/uk-markal (accessed 5.30.21).

UKERC, 2009. Making the transition to a secure and low carbon energy system: synthesis report of the Energy 2050 project. UKERC, London.

UKERC Energy Data Centre, 2021. Centre for Energy Systems Integration [WWW Document]. URL https://ukerc.rl.ac.uk/cgi-bin/ercri5. 
pl? GChoose $=$ gregsum $\&$ GRN $=\mathrm{EP} / \mathrm{P} 001173 / 1 \&$ GrantRegion $=10 \&$ GrantOr $\mathrm{g}=109 \& H T C=361 \mathrm{DDE} 2 \&$ SHTC $=80680 \mathrm{D}($ accessed 5.30.21).

van der Vleuten, E., 2004. Infrastructures and societal change. A view from the large technical systems field. Technology Analysis and Strategic Management 16, 395-414. https://doi.org/10.1080/0953732042000251160

Wheatcroft, E., Wynn, H., Dent, C.J., Smith, J.Q., Copeland, C.L., Ralph, D., Zachary, S., 2019. The Scenario Culture. https://arxiv.org/abs/1911.13170. Winskel, M., Ketsopoulou Irina, Churchhouse, T., 2015. UKERC interdisciplinary review. UKERC, London.

Open Access This chapter is licensed under the terms of the Creative Commons Attribution 4.0 International License (http://creativecommons.org/licenses/ by $/ 4.0 /$ ), which permits use, sharing, adaptation, distribution and reproduction in any medium or format, as long as you give appropriate credit to the original author(s) and the source, provide a link to the Creative Commons licence and indicate if changes were made.

The images or other third party material in this chapter are included in the chapter's Creative Commons licence, unless indicated otherwise in a credit line to the material. If material is not included in the chapter's Creative Commons licence and your intended use is not permitted by statutory regulation or exceeds the permitted use, you will need to obtain permission directly from the copyright holder.

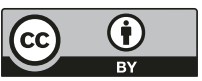

